\title{
Efficient Generalized Ratio-Product Type Estimators for Finite Population Mean with Ranked Set Sampling
}

\author{
V. L. Mandowara and Nitu Mehta (Ranka) \\ M. L. Sukhadia University, Udaipur, Rajasthan, India
}

\begin{abstract}
In this paper we suggest two modified estimators of the population mean using the power transformation based on ranked set sampling (RSS). The first order approximation of the bias and of the mean squared error of the proposed estimators are obtained. A generalized version of the suggested estimators by applying the power transformation is also presented. Theoretically, it is shown that these suggested estimators are more efficient than the estimators in simple random sampling (SRS). A numerical illustration is also carried out to demonstrate the merits of the proposed estimators using RSS over the usual estimators in SRS.
\end{abstract}

Zusammenfassung: In dieser Arbeit schlagen wir zwei modifizierte Schätzer für das Populationsmittel vor, indem wir die Power-Transformation basierend auf "Ranked Set Sampling" (RSS) verwenden. Approximationen erster Ordnung für den Bias und den mittleren quadratischen Fehlers der vorgeschlagenen Schätzer werden erhalten. Eine verallgemeinerte Version der vorgeschlagenen Schätzer durch die Anwendung der Power-Transformation wird auch vorgestellt. Theoretisch wird gezeigt, dass diese vorgeschlagenen Schätzer effizienter sind als die Schätzer unter "Simple Random Sampling" (SRS). Eine numerische Darstellung wird auch durchgeführt, um die Vorzüge dieser Schätzer unter RSS über die üblichen Schätzer unter SRS aufzuzeigen.

Keywords: Ranked Set Sampling, Ratio Estimator, Power Transformation Estimator, Auxiliary Variable, Coefficient of Variation, Coefficient of Kurtosis.

\section{Introduction}

The literature on ranked set sampling describes a great variety of techniques for using auxiliary information to obtain more efficient estimators. Ranked set sampling was first suggested by McIntyre (1952) to increase the efficiency of estimator of population mean. Kadilar, Unyazici, and Cingi (2009) used this technique to improve ratio estimator given by Prasad (1989). Here we shall propose two modified estimators of population mean using power transformation using RSS based on auxiliary variable.

The classical ratio estimators given by Cochran (1940) for estimating the population mean $\bar{Y}$ is defined as

$$
\bar{y}_{R}=\bar{y}\left(\frac{\bar{X}}{\bar{x}}\right)
$$

where $\bar{y}$ is the sample mean for study variable $y$ and $\bar{x}, \bar{X}$ are the sample mean and population mean, respectively, for the auxiliary variable $x$. 
When the population coefficient of variation $C_{x}$ of the auxiliary variable $x$ is known, Sisodia and Dwivedi (1981) give a modified ratio estimator for $\bar{Y}$ as

$$
\bar{y}_{S D}=\bar{y}\left(\frac{\bar{X}+C_{x}}{\bar{x}+C_{x}}\right) .
$$

Motivated by Sisodia and Dwivedi (1981), H. P. Singh and Kakran (1993) developed a ratio-type estimator for $\bar{Y}$ as

$$
\bar{y}_{S K}=\bar{y}\left(\frac{\bar{X}+\beta_{2}(x)}{\bar{x}+\beta_{2}(x)}\right),
$$

where $\beta_{2}(x)$ is the known value of the coefficient of kurtosis of an auxiliary variable $x$.

Utilizing the information on the coefficient of variation $C_{x}$ and the coefficient of kurtosis $\beta_{2}(x)$ of the auxiliary variable $x$, Upadhyaya and Singh (1999) suggested the following ratio type estimators

$$
\begin{aligned}
& \bar{y}_{U P 1}=\bar{y}\left(\frac{\bar{X} \beta_{2}(x)+C_{x}}{\bar{x} \beta_{2}(x)+C_{x}}\right), \\
& \bar{y}_{U P 2}=\bar{y}\left(\frac{\bar{X} C_{x}+\beta_{2}(x)}{\bar{x} C_{x}+\beta_{2}(x)}\right) .
\end{aligned}
$$

By applying the power transformation on the Upadhyaya and Singh (1999) estimators, H. P. Singh, Tailor, Singh, and Kim (2008) suggested modified estimators as

$$
\begin{aligned}
& \bar{y}_{U P 1(\alpha)}=\bar{y}\left(\frac{\bar{X} \beta_{2}(x)+C_{x}}{\bar{x} \beta_{2}(x)+C_{x}}\right)^{\alpha} \\
& \bar{y}_{U P 2(\delta)}=\bar{y}\left(\frac{\bar{X} C_{x}+\beta_{2}(x)}{\bar{x} C_{x}+\beta_{2}(x)}\right)^{\delta},
\end{aligned}
$$

where $\alpha$ and $\delta$ are suitably chosen scalars such that the mean squared errors of $\bar{y}_{U P 1(\alpha)}$ and $\bar{y}_{U P 2(\delta)}$ are minimum. are

To the first degree of approximation, the mean squared error (MSE) of these estimators

$$
\begin{aligned}
\operatorname{MSE}\left(\bar{y}_{R}\right) & =\frac{1}{n} \bar{Y}^{2}\left(C_{y}^{2}+C_{x}^{2}-2 \rho_{y x} C_{y} C_{x}\right), \\
\operatorname{MSE}\left(\bar{y}_{S D}\right) & =\frac{1}{n} \bar{Y}^{2}\left(C_{y}^{2}+\lambda_{1}^{2} C_{x}^{2}-2 \lambda_{1} \rho_{y x} C_{y} C_{x}\right), \\
\operatorname{MSE}\left(\bar{y}_{S K}\right) & =\frac{1}{n} \bar{Y}^{2}\left(C_{y}^{2}+\lambda_{2}^{2} C_{x}^{2}-2 \lambda_{2} \rho_{y x} C_{y} C_{x}\right), \\
\operatorname{MSE}\left(\bar{y}_{U P 1}\right) & =\frac{1}{n} \bar{Y}^{2}\left(C_{y}^{2}+\gamma_{1}^{2} C_{x}^{2}-2 \gamma_{1} \rho_{y x} C_{y} C_{x}\right), \\
\operatorname{MSE}\left(\bar{y}_{U P 2}\right) & =\frac{1}{n} \bar{Y}^{2}\left(C_{y}^{2}+\gamma_{2}^{2} C_{x}^{2}-2 \gamma_{2} \rho_{y x} C_{y} C_{x}\right), \\
\operatorname{MSE}\left(\bar{y}_{U P 1(\alpha)}\right) & =\frac{1}{n} \bar{Y}^{2}\left(C_{y}^{2}+\phi_{1}^{2} \alpha^{2} C_{x}^{2}-2 \phi_{1} \alpha \rho_{y x} C_{y} C_{x}\right), \\
\operatorname{MSE}\left(\bar{y}_{U P 2(\delta)}\right) & =\frac{1}{n} \bar{Y}^{2}\left(C_{y}^{2}+\phi_{2}^{2} \delta^{2} C_{x}^{2}-2 \phi_{2} \delta \rho_{y x} C_{y} C_{x}\right),
\end{aligned}
$$


with

$$
\begin{aligned}
& C_{y}=\frac{S_{y}}{\bar{Y}}, \quad C_{x}=\frac{S_{x}}{\bar{X}}, \quad \lambda_{1}=\frac{\bar{X}}{\bar{X}+C_{x}}, \quad \lambda_{2}=\frac{\bar{X}}{\bar{X}+\beta_{2}(x)}, \\
& \gamma_{1}=\phi_{1}=\frac{\bar{X} \beta_{2}(x)}{\bar{X} \beta_{2}(x)+C_{x}}, \quad \gamma_{2}=\phi_{2}=\frac{\bar{X} C_{x}}{\bar{X} C_{x}+\beta_{2}(x)}, \quad \rho_{y x}=\frac{S_{y x}}{S_{y} S_{x}},
\end{aligned}
$$

where $\rho_{y x}$ denotes the correlation coefficient between $y$ and $x$.

\section{Ratio Estimator in Ranked Set Sampling}

In ranked set sampling (RSS) $m$ independent random sets are chosen (each of size $m$ ) and the units in each set are selected with equal probability and without replacement from a finite population of size $N$. The members of each random set are ranked with respect to the characteristic of the study variable or auxiliary variable. Then, the smallest unit is selected from the first ordered set and the second smallest unit is selected from the second ordered set. By this way, this procedure is continued until the unit with the largest rank is chosen from the $m$-th set. This cycle may be repeated $r$ times, so $m r=n$ units have been measured during this process. Thus, RSS and SRS have equivalent sample sizes $n$ for comparison of their biases and efficiencies.

When we rank on the auxiliary variable, let $\left(y_{[i]}, x_{(i)}\right)$ denote the $i$-th judgment ordering of the study variable and the $i$-th perfect ordering for the auxiliary variable in the $i$-th set, where $i=1, \ldots, m$.

Samawi and Muttlak (1996) define the ratio estimator for the population mean as

$$
\bar{y}_{R, R S S}=\bar{y}_{[n]}\left(\frac{\bar{X}}{\bar{x}_{(n)}}\right),
$$

where

$$
\bar{y}_{[n]}=\frac{1}{n} \sum_{i=1}^{n} y_{[i]}, \quad \bar{x}_{(n)}=\frac{1}{n} \sum_{i=1}^{n} x_{(i)}
$$

are the ranked set sample means for the variables $y$ and $x$, respectively.

To the first degree of approximation, the MSE of the estimator $\bar{y}_{R, R S S}$ is given by (after ignoring the finite population correction factor)

$$
\operatorname{MSE}\left(\bar{y}_{R, R S S}\right)=\bar{Y}^{2}\left(\frac{1}{m r}\left(C_{y}^{2}+C_{x}^{2}-2 \rho_{y x} C_{y} C_{x}\right)-\left(W_{y[i]}-W_{x(i)}\right)^{2}\right)
$$

where

$$
W_{x(i)}^{2}=\frac{1}{m^{2} r} \frac{1}{\bar{X}^{2}} \sum_{i=1}^{m} \tau_{x(i)}^{2}, \quad W_{y[i]}^{2}=\frac{1}{m^{2} r} \frac{1}{\bar{Y}^{2}} \sum_{i=1}^{m} \tau_{y[i]}^{2}
$$

with

$$
\tau_{x(i)}=\mu_{x(i)}-\bar{X}, \quad \tau_{y[i]}=\mu_{y[i]}-\bar{Y}
$$




\section{Suggested Estimators Based on Ranked Set Sampling}

Motivated by Sisodia and Dwivedi (1981) we suggest a ratio-type estimator for $\bar{Y}$ using RSS, when the population coefficient of variation of the auxiliary variable $C_{x}$ is known as

$$
\bar{y}_{R S S, M M 1}=\bar{y}_{[n]}\left(\frac{\bar{X}+C_{x}}{\bar{x}_{(n)}+C_{x}}\right) .
$$

To obtain the bias and the MSE of this estimator we put $\bar{y}_{[n]}=\bar{Y}\left(1+\varepsilon_{0}\right)$ and $\bar{x}_{(n)}=$ $\bar{X}\left(1+\varepsilon_{1}\right)$ so that $\mathrm{E}\left(\varepsilon_{0}\right)=\mathrm{E}\left(\varepsilon_{1}\right)=0$ as $\mathrm{E}\left(\bar{y}_{[n]}\right)=\bar{Y}$ and $\mathrm{E}\left(\bar{x}_{(n)}\right)=\bar{X}$ under ranked set sampling. Moreover,

$$
\begin{aligned}
\operatorname{var}\left(\varepsilon_{0}\right) & =\mathrm{E}\left(\varepsilon_{0}^{2}\right)=\frac{\operatorname{var}\left(\bar{y}_{[n]}\right)}{\bar{Y}^{2}}=\frac{1}{m r} \frac{1}{\bar{Y}^{2}}\left(S_{y}^{2}-\frac{1}{m} \sum_{i=1}^{m} \tau_{y[i]}^{2}\right) \\
& =\theta C_{y}^{2}-W_{y[i]}^{2} \quad \text { with } \quad \theta=\frac{1}{m r}
\end{aligned}
$$

and similarly

$$
\operatorname{var}\left(\varepsilon_{1}\right)=\mathrm{E}\left(\varepsilon_{1}^{2}\right)=\theta C_{x}^{2}-W_{x(i)}^{2}
$$

and

$$
\begin{aligned}
\operatorname{cov}\left(\varepsilon_{0}, \varepsilon_{1}\right) & =\mathrm{E}\left(\varepsilon_{0} \varepsilon_{1}\right)=\frac{\operatorname{cov}\left(\bar{y}_{[n]}, \bar{x}_{(n)}\right)}{\overline{X Y}}=\frac{1}{\overline{X Y}} \frac{1}{m r}\left(S_{y x}-\frac{1}{m} \sum_{i=1}^{m} \tau_{y x(i)}\right) \\
& =\theta \rho_{y x} C_{y} C_{x}-W_{y x(i)},
\end{aligned}
$$

where

$$
W_{y x(i)}=\frac{1}{m^{2} r} \frac{1}{\overline{X Y}} \sum_{i=1}^{m} \tau_{y x(i)} \quad \text { with } \quad \tau_{y x(i)}=\left(\mu_{y[i]}-\bar{Y}\right)\left(\mu_{x(i)}-\bar{X}\right) .
$$

Further to validate the first degree of approximation, we assume that the sample size is large enough to get $\left|\varepsilon_{0}\right|$ and $\left|\varepsilon_{1}\right|$ as small as possible such that the terms involving $\varepsilon_{0}$ or $\varepsilon_{1}$ in a degree greater than two are negligible.

The bias and the MSE of $\bar{y}_{R S S, M M 1}$ are found next. Since

$$
\operatorname{bias}\left(\bar{y}_{R S S, M M 1}\right)=\mathrm{E}\left(\bar{y}_{R S S, M M 1}\right)-\bar{Y}
$$

and

$$
\bar{y}_{R S S, M M 1}=\bar{Y}\left(1+\varepsilon_{0}\right)\left(1+\lambda_{1} \varepsilon_{1}\right)^{-1}, \quad \lambda_{1}=\frac{\bar{X}}{\bar{X}+C_{x}}
$$

we suppose $\left|\lambda_{1} \varepsilon_{1}\right|<1$ so that $\left(1+\lambda_{1} \varepsilon_{1}\right)^{-1}$ is expandable and derive

$$
\bar{y}_{R S S, M M 1}=\bar{Y}\left(1+\varepsilon_{0}\right)\left\{1-\lambda_{1} \varepsilon_{1}+\lambda_{1}^{2} \varepsilon_{1}^{2}+O\left(\lambda_{1} \varepsilon_{1}\right)\right\}
$$

Because $\mathrm{E}\left(\varepsilon_{0}\right)=\mathrm{E}\left(\varepsilon_{1}\right)=0$ we have

$$
\begin{aligned}
\operatorname{bias}\left(\bar{y}_{R S S, M M 1}\right) & =\bar{Y}\left(\lambda_{1}^{2} \mathrm{E}\left(\varepsilon_{1}^{2}\right)-\lambda_{1} \mathrm{E}\left(\varepsilon_{0} \varepsilon_{1}\right)\right) \\
& =\bar{Y}\left(\lambda_{1}^{2}\left\{\theta C_{x}^{2}-W_{x(i)}^{2}\right\}-\lambda_{1}\left\{\theta \rho_{y x} C_{y} C_{x}-W_{y x(i)}\right\}\right) \\
& =\bar{Y}\left(\theta\left(\lambda_{1}^{2} C_{x}^{2}-\lambda_{1} \rho_{y x} C_{y} C_{x}\right)-\left\{\lambda_{1}^{2} W_{x(i)}^{2}-\lambda_{1} W_{y x(i)}\right\}\right) .
\end{aligned}
$$


Now

$$
\begin{aligned}
\operatorname{MSE}\left(\bar{y}_{R S S, M M 1}\right) & =\mathrm{E}\left(\bar{y}_{R S S, M M 1}-\bar{Y}\right)^{2} \\
& =\bar{Y}^{2} \mathrm{E}\left(\varepsilon_{0}-\lambda_{1} \varepsilon_{1}+\lambda_{1}^{2} \varepsilon_{1}^{2}-2 \lambda_{1} \varepsilon_{0} \varepsilon_{1}\right)^{2} \\
& =\bar{Y}^{2} \mathrm{E}\left(\varepsilon_{0}^{2}+\lambda_{1}^{2} \varepsilon_{1}^{2}-2 \lambda_{1} \varepsilon_{0} \varepsilon_{1}\right) \\
& =\bar{Y}^{2}\left(\theta C_{y}^{2}-W_{y[i]}^{2}+\lambda_{1}^{2}\left(\theta C_{x}^{2}-W_{x(i)}^{2}\right)-2 \lambda_{1}\left(\theta \rho_{y x} C_{y} C_{x}-W_{y x(i)}\right)\right) \\
& =\bar{Y}^{2}\left(\theta\left\{C_{y}^{2}+\lambda_{1}^{2} C_{x}^{2}-2 \lambda_{1} \rho_{y x} C_{y} C_{x}\right\}-\left\{W_{y[i]}^{2}+\lambda_{1}^{2} W_{x(i)}^{2}-2 \lambda_{1} W_{y x(i)}\right\}\right) \\
& =\bar{Y}^{2}\left(\theta\left\{C_{y}^{2}+\lambda_{1}^{2} C_{x}^{2}-2 \lambda_{1} \rho_{y x} C_{y} C_{x}\right\}-\left\{W_{y[i]}-\lambda_{1} W_{x(i)}\right\}^{2}\right) .
\end{aligned}
$$

Motivated by H. P. Singh and Kakran (1993) we suggest another new ratio estimator in ranked set sampling as

$$
\bar{y}_{R S S, M M 2}=\bar{y}_{[n]}\left(\frac{\bar{X}+\beta_{2}(x)}{\bar{x}_{(n)}+\beta_{2}(x)}\right) .
$$

Similarly, the bias and the mean squared error are obtained, respectively, as

$$
\operatorname{bias}\left(\bar{y}_{R S S, M M 2}\right)=\bar{Y}\left(\theta\left(\lambda_{2}^{2} C_{x}^{2}-\lambda_{2} \rho_{y x} C_{y} C_{x}\right)-\left\{\lambda_{2}^{2} W_{x(i)}^{2}-\lambda_{2} W_{y x(i)}\right\}\right)
$$

and

$$
\operatorname{MSE}\left(\bar{y}_{R S S, M M 2}\right)=\bar{Y}^{2}\left(\theta\left\{C_{y}^{2}+\lambda_{2}^{2} C_{x}^{2}-2 \lambda_{2} \rho_{y x} C_{y} C_{x}\right\}-\left\{W_{y[i]}-\lambda_{2} W_{x(i)}\right\}^{2}\right) .
$$

Motivated by Upadhyaya and Singh (1999) we also proposed two more ratio type estimators considering both coefficients of variation and kurtosis using ranked set sampling as

$$
\begin{aligned}
& \bar{y}_{R S S, M M 3}=\bar{y}_{[n]}\left(\frac{\bar{X} \beta_{2}(x)+C_{x}}{\bar{x}_{(n)} \beta_{2}(x)+C_{x}}\right) \\
& \bar{y}_{R S S, M M 4}=\bar{y}_{[n]}\left(\frac{\bar{X} C_{x}+\beta_{2}(x)}{\bar{x}_{(n)} C_{x}+\beta_{2}(x)}\right) .
\end{aligned}
$$

The bias and the MSE of $\bar{y}_{R S S, M M 3}$ can be found as follows. Since

$$
\operatorname{bias}\left(\bar{y}_{R S S, M M 3}\right)=\mathrm{E}\left(\bar{y}_{R S S, M M 3}\right)-\bar{Y}
$$

and

$$
\bar{y}_{R S S, M M 3}=\bar{Y}\left(1+\varepsilon_{0}\right)\left(1+\gamma_{1} \varepsilon_{1}\right)^{-1}, \quad \gamma_{1}=\frac{\bar{X} \beta_{2}(x)}{\bar{X} \beta_{2}(x)+C_{x}},
$$

we suppose that $\left|\gamma_{1} \varepsilon_{1}\right|<1$ such that $\left(1+\gamma_{1} \varepsilon_{1}\right)^{-1}$ is expandable and get

$$
\bar{y}_{R S S, M M 3}=\bar{Y}\left(1+\varepsilon_{0}\right)\left\{1-\gamma_{1} \varepsilon_{1}+\gamma_{1}^{2} \varepsilon_{1}^{2}+O\left(\gamma_{1} \varepsilon_{1}\right)\right\} \text {. }
$$

Therefore,

$$
\begin{aligned}
\operatorname{bias}\left(\bar{y}_{R S S, M M 3}\right) & =\bar{Y}\left(\lambda^{2} \mathrm{E}\left(\varepsilon_{1}^{2}\right)-\lambda \mathrm{E}\left(\varepsilon_{0} \varepsilon_{1}\right)\right) \\
& =\bar{Y}\left(\gamma_{1}^{2}\left\{\theta C_{x}^{2}-W_{x(i)}^{2}\right\}-\gamma_{1}\left\{\theta \rho_{y x} C_{y} C_{x}-W_{y x(i)}\right\}\right) \\
& =\bar{Y}\left(\theta\left(\gamma_{1}^{2} C_{x}^{2}-\gamma_{1} \rho_{y x} C_{y} C_{x}\right)-\left\{\gamma_{1}^{2} W_{x(i)}^{2}-\gamma_{1} W_{y x(i)}\right\}\right)
\end{aligned}
$$


and

$$
\begin{aligned}
\operatorname{MSE}\left(\bar{y}_{R S S, M M 3}\right) & =\mathrm{E}\left(\bar{y}_{R S S, M M 3}-\bar{Y}\right)^{2} \\
& =\bar{Y}^{2} \mathrm{E}\left(\varepsilon_{0}-\gamma_{1} \varepsilon_{1}+\gamma_{1}^{2} \varepsilon_{1}^{2}-2 \gamma_{1} \varepsilon_{0} \varepsilon_{1}\right)^{2} \\
& =\bar{Y}^{2} \mathrm{E}\left(\varepsilon_{0}^{2}+\gamma_{1}^{2} \varepsilon_{1}^{2}-2 \gamma_{1} \varepsilon_{0} \varepsilon_{1}\right) \\
& =\bar{Y}^{2}\left(\theta C_{y}^{2}-W_{y[i]}^{2}+\lambda^{2}\left(\theta C_{x}^{2}-W_{x(i)}^{2}\right)-2 \lambda\left(\theta \rho_{y x} C_{y} C_{x}-W_{y x(i)}\right)\right) \\
& =\bar{Y}^{2}\left(\theta\left\{C_{y}^{2}+\gamma_{1}^{2} C_{x}^{2}-2 \gamma_{1} \rho_{y x} C_{y} C_{x}\right\}-\left\{W_{y[i]}^{2}+\gamma_{1}^{2} W_{x(i)}^{2}-2 \gamma_{1} W_{y x(i)}\right\}\right) \\
& =\bar{Y}^{2}\left(\theta\left\{C_{y}^{2}+\gamma_{1}^{2} C_{x}^{2}-2 \gamma_{1} \rho_{y x} C_{y} C_{x}\right\}-\left\{W_{y[i]}-\gamma_{1} W_{x(i)}\right\}^{2}\right) .
\end{aligned}
$$

Similarly, the bias and mean squared error of the estimator $\bar{y}_{R S S, M M 4}$ can be obtained, respectively, by changing the place of coefficient of kurtosis and coefficient of variation, as

$$
\operatorname{bias}\left(\bar{y}_{R S S, M M 4}\right)=\bar{Y}\left(\theta\left(\gamma_{2}^{2} C_{x}^{2}-\gamma_{2} \rho_{y x} C_{y} C_{x}\right)-\left\{\gamma_{2}^{2} W_{x(i)}^{2}-\gamma_{2} W_{y x(i)}\right\}\right)
$$

and

$$
\operatorname{MSE}\left(\bar{y}_{R S S, M M 4}\right)=\bar{Y}^{2}\left(\theta\left\{C_{y}^{2}+\gamma_{2}^{2} C_{x}^{2}-2 \gamma_{2} \rho_{y x} C_{y} C_{x}\right\}-\left\{W_{y[i]}-\gamma_{2} W_{x(i)}\right\}^{2}\right) .
$$

By applying the power transformation on $\bar{y}_{R S S, M M 3}$ and $\bar{y}_{R S S, M M 4}$ given in (18) and (19), we now propose generalized estimators as

$$
\begin{aligned}
& \bar{y}_{R S S, M M 3(\alpha)}=\bar{y}_{[n]}\left(\frac{\bar{X} \beta_{2}(x)+C_{x}}{\bar{x}_{(n)} \beta_{2}(x)+C_{x}}\right)^{\alpha} \\
& \bar{y}_{R S S, M M 4(\delta)}=\bar{y}_{[n]}\left(\frac{\bar{X} C_{x}+\beta_{2}(x)}{\bar{x}_{(n)} C_{x}+\beta_{2}(x)}\right)^{\delta} .
\end{aligned}
$$

The bias and MSE of the estimator $\bar{y}_{R S S, M M 3(\alpha)}$ to the first degree of approximation, are obtained next. We have

$$
\operatorname{bias}\left(\bar{y}_{R S S, M M 3(\alpha)}\right)=\mathrm{E}\left(\bar{y}_{R S S, M M 3(\alpha)}\right)-\bar{Y}
$$

where

$$
\begin{aligned}
\bar{y}_{R S S, M M 3(\alpha)} & =\bar{Y}\left(1+\varepsilon_{0}\right)\left(1+\phi_{1} \varepsilon_{1}\right)^{-\alpha} \\
& =\bar{Y}\left(\left(1+\varepsilon_{0}\right)\left\{1-\phi_{1} \alpha \varepsilon_{1}+\frac{\alpha(\alpha+1)}{2} \phi_{1}^{2} \varepsilon_{1}^{2}+O\left(\varepsilon_{1}\right)\right\}\right)
\end{aligned}
$$

Thus

$$
\begin{aligned}
\operatorname{bias}\left(\bar{y}_{R S S, M M 3(\alpha)}\right) & =\bar{Y}\left(\phi_{1}^{2} \frac{\alpha(\alpha+1)}{2}\left\{\theta C_{x}^{2}-W_{x(i)}^{2}\right\}-\phi_{1} \alpha\left\{\theta \rho_{y x} C_{y} C_{x}-W_{y x(i)}\right\}\right) \\
& =\frac{\bar{Y}}{2}\left(\phi_{1} \alpha \theta\left\{(\alpha+1) C_{x}^{2}-2 \rho_{y x} C_{y} C_{x}\right\}-\phi_{1} \alpha\left\{(\alpha+1) W_{x(i)}^{2}-2 W_{y x(i)}\right\}\right)
\end{aligned}
$$


and

$$
\begin{aligned}
\operatorname{MSE}\left(\bar{y}_{R S S, M M 3(\alpha)}\right) & =\mathrm{E}\left(\bar{y}_{R S S, M M 3(\alpha)}-\bar{Y}\right)^{2} \\
& =\bar{Y}^{2} \mathrm{E}\left(\varepsilon_{0}-\phi_{1} \alpha \varepsilon_{1}+\phi_{1}^{2} \frac{\alpha(\alpha+1)}{2} \varepsilon_{1}^{2}-\phi_{1} \alpha \varepsilon_{0} \varepsilon_{1}\right)^{2} \\
& =\bar{Y}^{2} \mathrm{E}\left(\varepsilon_{0}^{2}+\phi_{1}^{2} \alpha^{2} \varepsilon_{1}^{2}-2 \phi_{1} \alpha \varepsilon_{0} \varepsilon_{1}\right) \\
& =\bar{Y}^{2}\left(\theta C_{y}^{2}-W_{y[i]}^{2}+\phi_{1}^{2} \alpha^{2}\left(\theta C_{x}^{2}-W_{x(i)}^{2}\right)-2 \phi_{1} \alpha\left(\theta \rho_{y x} C_{y} C_{x}-W_{y x(i)}\right)\right) \\
& =\bar{Y}^{2}\left(\theta\left\{C_{y}^{2}+\alpha^{2} \phi_{1}^{2} C_{x}^{2}-2 \alpha \phi_{1} \rho_{y x} C_{y} C_{x}\right\}-\left\{W_{y[i]}-\phi_{1} \alpha W_{x(i)}\right\}^{2}\right) .(24)
\end{aligned}
$$

Similarly, the bias and mean squared error of the estimator $\bar{y}_{R S S, M M 4(\delta)}$ can be obtained, respectively, by changing the place of coefficient of kurtosis and coefficient of variation, as

$$
\operatorname{bias}\left(\bar{y}_{R S S, M M 4(\delta)}\right)=\frac{\bar{Y}}{2}\left(\phi_{2} \delta \theta\left\{(\delta+1) C_{x}^{2}-2 \rho_{y x} C_{y} C_{x}\right\}-\phi_{2} \delta\left\{(\delta+1) W_{x(i)}^{2}-2 W_{y x(i)}\right\}\right)
$$

and

$$
\operatorname{MSE}\left(\bar{y}_{R S S, M M 4(\delta)}\right)=\bar{Y}^{2}\left(\theta\left\{C_{y}^{2}+\delta^{2} \phi_{2}^{2} C_{x}^{2}-2 \delta \phi_{2} \rho_{y x} C_{y} C_{x}\right\}-\left\{W_{y[i]}-\phi_{2} \delta W_{x(i)}\right\}^{2}\right)
$$

\section{Optimality of $\alpha$ and $\delta$}

The optimum value of $\alpha$ to minimize the MSE of $\bar{y}_{R S S, M M 3(\alpha)}$ can easily be found as zero of its derivative, i.e.

$$
\frac{\partial}{\partial \alpha} \operatorname{MSE}\left(\bar{y}_{R S S, M M 3(\alpha)}\right)=0
$$

which results in

$$
\alpha=\frac{\theta \rho_{x y} C_{x} C_{y}-W_{y x(i)}}{\phi_{1}\left(\theta C_{x}^{2}-W_{x(i)}^{2}\right)}
$$

and because $\operatorname{cov}\left(\bar{x}_{(n)}, \bar{y}_{[n]}\right)=\beta \operatorname{var}\left(\bar{x}_{(n)}\right)$ this is equivalent to

$$
\alpha=\rho_{x y} \frac{C_{y}}{\phi_{1} C_{x}} .
$$

Similarly,

$$
\delta=\rho_{x y} \frac{C_{y}}{\phi_{2} C_{x}} .
$$

After substituting (26) and (27), respectively, in (24) and (25), we obtain the minimum mean squared error of the proposed estimators as

$$
\begin{aligned}
\min \operatorname{MSE}\left(\bar{y}_{R S S, M M 3(\alpha)}\right) & =\min \operatorname{MSE}\left(\bar{y}_{R S S, M M 4(\delta)}\right) \\
& =\bar{Y}^{2}\left(\theta C_{y}^{2}\left(1-\rho_{y x}^{2}\right)-\left\{W_{y[i]}-K W_{x(i)}\right\}^{2}\right),
\end{aligned}
$$

where $K=\rho \frac{C_{y}}{C_{x}}$. 


\section{Efficiency Comparison}

On comparing (7) to (12) with (15), (17), (20), (21), (24) and (25), respectively, we obtain

1. $\operatorname{MSE}\left(\bar{y}_{S D}\right)-\operatorname{MSE}\left(\bar{y}_{R S S, M M 1}\right)=A_{1} \geq 0$, where $A_{1}=\left[W_{y[i]}-\lambda_{1} W_{x(i)}\right]^{2}$ $\Rightarrow \operatorname{MSE}\left(\bar{y}_{R S S, M M 1}\right) \leq \operatorname{MSE}\left(\bar{y}_{S D}\right)$

2. $\operatorname{MSE}\left(\bar{y}_{S K}\right)-\operatorname{MSE}\left(\bar{y}_{R S S, M M 2}\right)=A_{2} \geq 0$, where $A_{2}=\left[W_{y[i]}-\lambda_{2} W_{x(i)}\right]^{2}$ $\Rightarrow \operatorname{MSE}\left(\bar{y}_{R S S, M M 2}\right) \leq \operatorname{MSE}\left(\bar{y}_{S K}\right)$

3. $\operatorname{MSE}\left(\bar{y}_{U P 1}\right)-\operatorname{MSE}\left(\bar{y}_{R S S, M M 3}\right)=A_{3} \geq 0$, where $A_{3}=\left[W_{y[i]}-\gamma_{1} W_{x(i)}\right]^{2}$ $\Rightarrow \operatorname{MSE}\left(\bar{y}_{R S S, M M 3}\right) \leq \operatorname{MSE}\left(\bar{y}_{U P 1}\right)$

4. $\operatorname{MSE}\left(\bar{y}_{U P 2}\right)-\operatorname{MSE}\left(\bar{y}_{R S S, M M 4}\right)=A_{4} \geq 0$, where $A_{4}=\left[W_{y[i]}-\gamma_{2} W_{x(i)}\right]^{2}$ $\Rightarrow \operatorname{MSE}\left(\bar{y}_{R S S, M M 4}\right) \leq \operatorname{MSE}\left(\bar{y}_{U P 2}\right)$

5. $\operatorname{MSE}\left(\bar{y}_{U P 1(\alpha)}\right)-\operatorname{MSE}\left(\bar{y}_{R S S, M M 3(\alpha)}\right)=A_{5} \geq 0$, where $A_{5}=\left[W_{y[i]}-\phi_{1} \alpha W_{x(i)}\right]^{2}$ $\Rightarrow \operatorname{MSE}\left(\bar{y}_{R S S, M M 3(\alpha)}\right) \leq \operatorname{MSE}\left(\bar{y}_{U P 1(\alpha)}\right)$

6. $\operatorname{MSE}\left(\bar{y}_{U P 2(\delta)}\right)-\operatorname{MSE}\left(\bar{y}_{R S S, M M 4(\delta)}\right)=A_{6} \geq 0$, where $A_{6}=\left[W_{y[i]}-\phi_{1} \delta W_{x(i)}\right]^{2}$ $\Rightarrow \operatorname{MSE}\left(\bar{y}_{R S S, M M 4(\delta)}\right) \leq \operatorname{MSE}\left(\bar{y}_{U P 2(\delta)}\right)$

It is easily seen that the MSE of the suggested estimators given in (14), (16), (18), (19), (22), and (23) are always smaller than those of the estimators given in (1) to (6), respectively, because $A_{1}, A_{2}, A_{3}, A_{4}, A_{5}$, and $A_{6}$ are all non-negative values. As a result, the proposed generalized estimators $\bar{y}_{R S S, M M 3(\alpha)}$ and $\bar{y}_{R S S, M M 4(\delta)}$ for the population mean using power transformation in ranked set sampling are more efficient than the usual estimators $\bar{y}_{U P 1(\alpha)}$ and $\bar{y}_{U P 2(\delta)}$.

For $\alpha=\delta=-1$, the generalized estimators given in (22) and (23) turn to product type estimators.

\section{Numerical Illustration}

To compare the efficiencies of the various estimators of our study, we take a population of size $N=50$ (see page 1111 in the appendix of S. Singh, 2003). The example considers the data of agricultural loans outstanding of all operating banks in different states of the USA in 1997, where $y$ is the real estate farm loans (study variable) in $1000 \$$ and $x$ is the non-real estate loans (auxiliary variable) in 1000 .

For the above population, the parameters are summarized as: $Y=27771.73, X=$ $43908.12, \bar{Y}=555.43, \bar{X}=878.16, S_{x}^{2}=1176526, S_{y}^{2}=342021.5, C_{x}^{2}=1.5256, C_{y}^{2}=$ $1.1086, \rho=0.8038, \beta_{2}(x)=1.9215, \lambda_{1}=0.9986, \lambda_{2}=0.9978, \gamma_{1}=\phi_{1}=0.9993, \gamma_{2}=$ $\phi_{2}=0.9982$, and $K=0.6852$.

From this population we have taken 100 ranked set samples with size $m=4$ and number of cycles $r=3$, so that $n=m r=12$. For these 100 ranked set samples chosen, we have computed estimated MSE's of the proposed estimators $\bar{y}_{M M 1, R S S}, \bar{y}_{M M 2, R S S}$, $\bar{y}_{M M 3, R S S}, \bar{y}_{M M 4, R S S}, \bar{y}_{R S S, M M 3(\alpha)}$, and $\bar{y}_{R S S, M M 4(\delta)}$ which are given in Table 2. Table 1 
Table 1: Estimated MSE's of various estimators using SRS (Note that $\bar{y}_{U P 1(\alpha)}=\bar{y}_{U P 2(\delta)}$ )

\begin{tabular}{|l|ccccc|}
\hline Estimator & $\bar{y}_{S D}$ & $\bar{y}_{S K}$ & $\bar{y}_{U P 1}$ & $\bar{y}_{U P 2}$ & $\bar{y}_{U P 1(\alpha)}$ \\
\hline MSE & 19936.6 & 19313.5 & 14006.0 & 13925.8 & 10088.0 \\
\hline
\end{tabular}

shows the MSE's of the estimators $\bar{y}_{S D}, \bar{y}_{S K}, \bar{y}_{U P 1}, \bar{y}_{U P 2}, \bar{y}_{U P 1(\alpha)}$, and $\bar{y}_{U P 2(\delta)}$, given in H. P. Singh et al. (2008).

\section{Conclusion}

On comparing Table 1 with Table 2 and Table 3 for the 100 ranked set samples, we see that the MSE's of the proposed estimators are smaller than those of the estimators given by previous authors. As a result, all the proposed new ratio type estimators $\bar{y}_{M M 1, R S S}$, $\bar{y}_{M M 2, R S S}, \bar{y}_{M M 3, R S S}, \bar{y}_{M M 4, R S S}, \bar{y}_{R S S, M M 3(\alpha)}$ and $\bar{y}_{R S S, M M 4(\delta)}$ for the population mean using RSS are more efficient than the respective estimators $\bar{y}_{S D}, \bar{y}_{S K}, \bar{y}_{U P 1}, \bar{y}_{U P 2}, \bar{y}_{U P 1(\alpha)}$ and $\bar{y}_{U P 2(\delta)}$ under SRS. Thus, if the coefficient of variation and the coefficient of kurtosis are known for the auxiliary variable, then these proposed estimators are recommended for use in practice.

\section{Acknowledgements}

The authors are thankful to the referees for their valuable suggestion.

\section{References}

Cochran, W. G. (1940). Some properties of estimators based on sampling scheme with varying probabilities. Australian Journal of Statistics, 17, 22-28.

Kadilar, C., Unyazici, Y., and Cingi, H. (2009). Ratio estimator for the population mean using ranked set sampling. Statistical Papers, 50, 301-309.

McIntyre, G. A. (1952). A method of unbiased selective sampling using ranked sets. Australian Journal of Agricultural Research, 3, 385-390.

Prasad, B. (1989). Some improved ratio type estimators of population mean and ratio in finite population sample surveys. Communications in Statatistics, Theory and Methods, 18, 379-392.

Samawi, H. M., and Muttlak, H. A. (1996). Estimation of ratio using rank set sampling. Biometrical Journal, 38, 753-764.

Singh, H. P., and Kakran, M. S. (1993). A modified ratio estimator using known coefficient of kurtosis of an auxiliary character.

Singh, H. P., Tailor, R., Singh, S., and Kim, J. M. (2008). A modified estimator of population mean using power transformation. Statistical Papers, 49, 37-58.

Singh, S. (2003). Advanced Sampling Theory with Applications, Volume II. Kluwer Academic Publishers. 
Table 2: Estimated MSE's of different new estimators using RSS. Note that $\bar{y}_{R S S, M M 3(\alpha)}=\bar{y}_{R S S, M M 4(\delta)}$

\begin{tabular}{|c|c|c|c|c|c|}
\hline Estimator & $\bar{y}_{R S S, M M 1}$ & $\bar{y}_{R S S, M M 2}$ & $\bar{y}_{R S S, M M 3}$ & $\bar{y}_{R S S, M M 4}$ & $\bar{y}_{R S S, M M 3(\alpha)}$ \\
\hline 1 & 12414.7 & 12400.0 & 12476.7 & 12408.1 & 10075.5 \\
\hline 2 & 12988.8 & 12971.6 & 13053.0 & 12981.0 & 10040.1 \\
\hline 3 & 13167.9 & 13149.8 & 13232.9 & 13159.7 & 10026.6 \\
\hline 4 & 13890.6 & 13868.7 & 13958.8 & 13880.4 & 9102.2 \\
\hline 5 & 13576.7 & 13550.1 & 13649.2 & 13564.2 & 7036.4 \\
\hline 6 & 11703.5 & 11671.2 & 11780.9 & 11688.1 & 2807.5 \\
\hline 7 & 12834.1 & 12817.5 & 12897.8 & 12826.6 & 10058.4 \\
\hline 8 & 10293.4 & 10286.5 & 10348.6 & 10290.7 & 10029.0 \\
\hline 9 & 13714.0 & 13687.2 & 13786.5 & 13701.3 & 6141.9 \\
\hline 10 & 13921.1 & 13897.2 & 13991.2 & 13909.9 & 8136.1 \\
\hline 11 & 11171.5 & 11164.6 & 11226.8 & 11168.8 & 10028.7 \\
\hline 12 & 13888.4 & 13864.4 & 13958.6 & 13877.2 & 9056.6 \\
\hline 13 & 10645.2 & 10635.0 & 10703.3 & 10640.9 & 9910.5 \\
\hline 14 & 13926.9 & 13904.4 & 13995.8 & 13916.4 & 9023.7 \\
\hline 15 & 13397.1 & 13370.7 & 13469.4 & 13384.7 & 7522.3 \\
\hline 16 & 13815.7 & 13795.5 & 13882.5 & 13806.3 & 8379.8 \\
\hline 17 & 13640.3 & 13615.1 & 13711.4 & 13628.4 & 8460.5 \\
\hline 18 & 13598.9 & 13579.8 & 13664.8 & 13590.1 & 9470.2 \\
\hline 19 & 13868.4 & 13846.8 & 13936.6 & 13858.4 & 9368.3 \\
\hline 20 & 13916.5 & 13894.1 & 13985.3 & 13906.1 & 9265.6 \\
\hline 21 & 11283.3 & 11250.6 & 11361.0 & 11267.7 & 2399.1 \\
\hline 22 & 13782.3 & 13761.0 & 13850.1 & 13772.4 & 9801.3 \\
\hline 23 & 13881.2 & 13859.5 & 13949.2 & 13871.1 & 9009.6 \\
\hline 24 & 13182.5 & 13165.6 & 13246.5 & 13174.8 & 9802.9 \\
\hline 25 & 10544.8 & 10537.9 & 10600.0 & 10542.1 & 9292.4 \\
\hline 26 & 13811.1 & 13790.2 & 13878.5 & 13801.4 & 9292.4 \\
\hline 27 & 13551.3 & 13533.2 & 13616.3 & 13543.0 & 9127.4 \\
\hline 28 & 13003.3 & 12987.2 & 13066.6 & 12996.1 & 9876.6 \\
\hline 29 & 8886.9 & 8880.4 & 8941.6 & 8884.4 & 9466.6 \\
\hline 30 & 13911.7 & 13887.4 & 13982.1 & 13900.3 & 7443.7 \\
\hline 31 & 10588.9 & 10577.6 & 10647.9 & 10584.0 & 9781.1 \\
\hline 32 & 12641.1 & 12625.7 & 12703.6 & 12634.2 & 10043.6 \\
\hline 33 & 11778.5 & 11765.6 & 11838.9 & 11772.8 & 10078.9 \\
\hline 34 & 12300.9 & 12285.2 & 12363.7 & 12293.8 & 10069.8 \\
\hline 35 & 13838.6 & 13814.0 & 13909.3 & 13827.1 & 8646.4 \\
\hline 36 & 11356.7 & 11347.0 & 11414.4 & 11352.7 & 10086.8 \\
\hline 37 & 13758.1 & 13737.9 & 13825.0 & 13748.8 & 9256.5 \\
\hline 38 & 13351.6 & 13332.7 & 13417.3 & 13342.8 & 9999.4 \\
\hline 39 & 13434.6 & 13416.8 & 13499.4 & 13426.5 & 9500.7 \\
\hline 40 & 13771.4 & 13745.3 & 13843.3 & 13759.1 & 6824.4 \\
\hline 41 & 12928.9 & 12912.7 & 12992.3 & 12921.6 & 9975.0 \\
\hline 42 & 12870.4 & 12851.9 & 12935.9 & 12861.9 & 10060.0 \\
\hline 43 & 12576.9 & 12563.8 & 12637.6 & 12571.1 & 9825.2 \\
\hline 44 & 13860.9 & 13839.0 & 13929.2 & 13850.7 & 9686.9 \\
\hline 45 & 12544.7 & 12531.5 & 12605.4 & 12538.9 & 9866.8 \\
\hline 46 & 13818.6 & 13793.1 & 13890.0 & 13806.6 & 7272.4 \\
\hline 47 & 13776.8 & 13756.7 & 13843.6 & 13767.5 & 8917.3 \\
\hline 48 & 9061.8 & 9058.5 & 9113.9 & 9060.9 & 9887.5 \\
\hline 49 & 12958.0 & 12929.7 & 13032.0 & 12944.6 & 5995.0 \\
\hline 50 & 13869.5 & 13848.5 & 13937.0 & 13859.7 & 8231.8 \\
\hline
\end{tabular}


Table 3: Estimated MSE's of different new estimators using RSS. Note that $\bar{y}_{R S S, M M 3(\alpha)}=\bar{y}_{R S S, M M 4(\delta)}$

\begin{tabular}{|c|c|c|c|c|c|}
\hline Estimator & $\bar{y}_{R S S, M M 1}$ & $\bar{y}_{R S S, M M 2}$ & $\bar{y}_{R S S, M M 3}$ & $\bar{y}_{R S S, M M 4}$ & $\bar{y}_{R S S, M M 3(\alpha)}$ \\
\hline 51 & 13357.4 & 13340.5 & 13421.3 & 13349.7 & 9365.7 \\
\hline 52 & 7661.9 & 7654.3 & 7717.7 & 7658.9 & 8419.4 \\
\hline 53 & 8235.4 & 8237.4 & 8282.7 & 8237.2 & 9983.6 \\
\hline 54 & 10449.1 & 10440.2 & 10506.0 & 10445.4 & 9947.6 \\
\hline 55 & 13073.1 & 13044.3 & 13147.5 & 13059.5 & 5575.9 \\
\hline 56 & 1806.8 & 1815.9 & 1848.0 & 1812.1 & 7294.8 \\
\hline 57 & 13933.8 & 13910.4 & 14003.4 & 13922.9 & 9173.3 \\
\hline 58 & 13677.3 & 13658.4 & 13743.1 & 13668.6 & 8846.3 \\
\hline 59 & 12227.8 & 12213.4 & 12289.6 & 12221.4 & 10088.0 \\
\hline 60 & 13366.9 & 13339.9 & 13439.7 & 13354.2 & 7012.6 \\
\hline 61 & 13674.2 & 13647.6 & 13746.6 & 13661.7 & 6768.0 \\
\hline 62 & 13926.8 & 13902.9 & 13996.8 & 13915.6 & 7530.2 \\
\hline 63 & 8494.8 & 8494.9 & 8543.9 & 8495.6 & 9941.9 \\
\hline 64 & 13017.1 & 12988.7 & 13091.1 & 13003.7 & 6003.9 \\
\hline 65 & 13875.4 & 13850.6 & 13946.3 & 13863.8 & 7538.5 \\
\hline 66 & 13377.5 & 13349.7 & 13450.9 & 13364.4 & 6305.2 \\
\hline 67 & 13931.9 & 13909.0 & 14001.1 & 13921.2 & 9662.0 \\
\hline 68 & 13688.4 & 13669.7 & 13754.0 & 13679.8 & 8571.0 \\
\hline 69 & 10301.1 & 10290.5 & 10359.6 & 10296.6 & 9706.8 \\
\hline 70 & 9995.6 & 9992.5 & 10047.5 & 9994.9 & 10088.0 \\
\hline 71 & 12745.8 & 12729.7 & 12809.0 & 12738.6 & 10055.7 \\
\hline 72 & 13884.8 & 13863.1 & 13952.8 & 13874.7 & 8934.0 \\
\hline 73 & 13850.5 & 13829.2 & 13918.3 & 13840.6 & 9174.8 \\
\hline 74 & 13925.9 & 13902.1 & 13995.9 & 13914.8 & 8154.6 \\
\hline 75 & 13586.8 & 13566.8 & 13653.5 & 13577.6 & 9883.3 \\
\hline 76 & 13834.9 & 13813.7 & 13902.6 & 13825.1 & 9282.6 \\
\hline 77 & 13141.6 & 13125.9 & 13204.4 & 13134.5 & 9522.3 \\
\hline 78 & 12733.2 & 12705.0 & 12807.1 & 12719.9 & 6059.3 \\
\hline 79 & 11764.0 & 11750.2 & 11825.2 & 11757.9 & 10035.3 \\
\hline 80 & 13869.0 & 13847.5 & 13937.0 & 13859.1 & 9120.3 \\
\hline 81 & 10669.4 & 10660.3 & 10726.5 & 10665.6 & 10005.9 \\
\hline 82 & 13093.9 & 13076.3 & 13158.4 & 13085.9 & 10010.7 \\
\hline 83 & 13618.9 & 13600.6 & 13684.1 & 13610.6 & 8857.9 \\
\hline 84 & 10486.9 & 10482.1 & 10540.3 & 10485.3 & 10082.6 \\
\hline 85 & 10540.6 & 10508.5 & 10617.9 & 10525.4 & 2269.6 \\
\hline 86 & 1755.0 & 1771.5 & 1789.7 & 1764.0 & 8492.2 \\
\hline 87 & 11823.1 & 11791.1 & 11900.2 & 11807.9 & 3092.4 \\
\hline 88 & 12753.5 & 12723.8 & 12828.6 & 12739.5 & 4973.8 \\
\hline 89 & 2060.9 & 2077.0 & 2096.0 & 2069.7 & 8607.7 \\
\hline 90 & 13925.9 & 13903.2 & 13994.8 & 13915.3 & 9375.5 \\
\hline 91 & 12665.4 & 12650.1 & 12727.9 & 12658.5 & 10032.8 \\
\hline 92 & 13634.5 & 13607.9 & 13706.9 & 13621.9 & 6949.3 \\
\hline 93 & 13935.8 & 13912.8 & 14005.0 & 13925.1 & 8337.9 \\
\hline 94 & 9761.7 & 9758.5 & 9813.7 & 9760.9 & 10070.1 \\
\hline 95 & 13854.0 & 13832.9 & 13921.6 & 13844.2 & 8828.4 \\
\hline 96 & 13926.6 & 13902.9 & 13996.5 & 13915.5 & 8781.9 \\
\hline 97 & 12045.1 & 12034.5 & 12103.5 & 12040.5 & 9916.0 \\
\hline 98 & 9633.5 & 9628.9 & 9686.7 & 9632.0 & 9984.5 \\
\hline 99 & 4198.5 & 4206.4 & 4240.8 & 4203.2 & 8710.8 \\
\hline 100 & 8512.7 & 8509.2 & 8565.0 & 8511.7 & 9623.5 \\
\hline
\end{tabular}


Sisodia, B. V. S., and Dwivedi, V. K. (1981). A modified ratio estimator using coefficient of variation of auxiliary variable. Journal of the Indian Society of Agricultural Statistics, 33, 13-18.

Upadhyaya, L. N., and Singh, H. P. (1999). Use of transformed auxiliary variable in estimating the finite population mean. Biometrical Journal, 41, 627-636.

Authors' address:

V. L. Mandowara and Nitu Mehta (Ranka)

Department of Mathematics and Statistics

University College of Science

M. L. Sukhadia University

Udaipur-313001

Rajasthan

India

E-mail: mandowara_vl@yahoo.co.in, nitumehta82@gmail.com 